\title{
Crecimiento inicial de Palo de Rosa (Aniba rosaeodora Ducke) en distintos ambientes de fertilidad
}

\author{
Wilmer Herrera VALENCIA ${ }^{1}$, Paulo de Tarso Barbosa SAMPAIO ${ }^{2}$, Luiz Augusto Gomes de SOUZA ${ }^{3}$
}

\begin{abstract}
RESUMEN
Los estudios de requerimientos nutricionales son de gran importancia para identificar aquellos más importantes en el desarrollo fisiológico y crecimiento de plántulas. Con el objetivo de evaluar las exigencias nutricionales y los efectos de la omisión de macronutrientes en el crecimiento de plántulas de Aniba rosaeodora; se realizó un experimento en el vivero del INPA-AmazonasBrasil teniendo como substrato un suelo Podozolico Rojo de baja disponibilidad de nutrientes. Se utilizaron 8 tratamientos bajo la técnica del nutriente faltante: Control (Suelo con macronutrientes), Suelo natural, y la omisión de un macronutriente por vez $(-\mathrm{N},-\mathrm{P},-\mathrm{K},-\mathrm{Ca},-\mathrm{Mg},-\mathrm{S})$. Se evaluaron las siguientes características: tasa de crecimiento relativo (TCR), Tasa de asimilación neta (TAN), peso de la materia seca de la parte aérea (MSPA) y de las raíces (MSR), contenido de nutrientes en las hojas, concluyendo que el $\mathrm{N}, \mathrm{Mg}$ y Ca, demostraron ser limitantes al crecimiento en suelo con pequeña disponibilidad; Las plántulas de $A$. rosaeodora presentaron un bajo requerimiento nutricional para el P, K y S. La omisión de Ca y N perjudica TCR de la especie. Los elementos más importantes para la MSPA fueron el Ca y el Mg; actuando el Mg más en el área foliar; por otro lado la omisión de azufre favorece la absorción de macronutrientes (N, $\mathrm{P}, \mathrm{K}, \mathrm{Ca}, \mathrm{Mg}$ ).
\end{abstract}

PALABRAS CLAVES: Floresta nativa, Estado nutricional, Macronutrientes, $A$. rosaeodora

\section{Growth of initial Rosewood (Aniba rosaeodora Ducke) in different environnement of fertility}

\begin{abstract}
Studies of nutritional requirements are of great importance for identifying the most important nutrients in physiologic development and seedling growth. A greenhouse pot experiment was conducted at INPA to evaluate the mineral nutritional demands and the effects of macronutrient omission in the plant growth of Aniba rosaeodora Ducke. The following treatments were used: Complete (fertilization with N, P, K, Ca, Mg, S, B and Zn), Standard (nature soil), Complete without N, Complete without P, Complete without $\mathrm{K}$, Complete without $\mathrm{Ca}$, Complete without $\mathrm{Mg}$, and Complete without $\mathrm{S}$. An Ultisoil with low nutrient availability was used as a substratum. The following characteristics were evaluated, relative growth rate (RGR), net assimilation rate (NAR), plant height, diameter, dry matter production of the aerial part (DMPAP) and amount of nutrient in the dry matter of leaves. We concluded from the results that: low availability of $\mathrm{N}, \mathrm{Ca}$ and $\mathrm{Mg}$ constraints the growth of the Aniba rosaeodora plants. Seedlings of $A$. rosaeodora required little P, K and S. The omission of Ca and $\mathrm{N}$ harmed the RGR of the species. The most important elements for DMPAP were $\mathrm{Ca}$ and $\mathrm{Mg}$; the $\mathrm{Mg}$ acting more in the leaf area; on the other hand, the omission of sulfur favored the macronutrient absorption ( $, \mathrm{P}, \mathrm{K}, \mathrm{Ca}, \mathrm{Mg})$.
\end{abstract}

KEY WORDS: Native forest, nutritional state, macronutrients, $A$. rosaeodora.

1 Universidade Federal do Para, E-mail: wilmer_br@yahoo.com.br

2 Instituto de Pesquisa da Amazônia, E-mail: sampaio@inpa.gov.br

${ }^{3}$ CPCA-INPA, E-mail: souzalag@inpa.gov.br 


\section{INTRODUCCIÓN}

El palo de rosa Aniba rosaeodora Ducke, es una especie de la familia Lauraceae de gran importancia económica en el mercado internacional debido al aceite esencial utilizado como fijador en la industria de perfumería (Sampaio, 1999). La demanda de este producto en el mercado nacional e internacional ha traído como consecuencia una sobreexplotación de la especie, decreciendo su población al punto que se ha incluido como una especie en amenaza de extinción en países como Colombia, Brasil y Surinam (IBAMA, 1992; Calderón, 1997; Werkhoven, 1997).

En vista del problema generado, los científicos se han dado a la tarea de desarrollar investigaciones, con miras a aumentar la supervivencia de la especie, sin embargo los resultados no han sido satisfactorios para incrementar la supervivencia y producción de plántulas debido a el crecimiento lento que presenta la especie. Según los resultados demostrados por investigadores como Viera, (1970), Carvalho, (1983), Alencar \& Fernández, (1978) y Sampaio, (1987), a pesar de la gran importancia de esta especie y de las investigaciones realizadas no hay suficiente información en el campo de la nutrición mineral, por lo cual se hace pertinente este tipo de estudio en la amazonía.

Uno de los grandes problemas de los plantíos de reforestación de palo de rosa es el desconocimiento de la autoecología de la especie especialmente en el aspecto de las exigencias nutricionales.

Se han observado diferencias entre las exigencias nutricionales de las especies forestales. En un experimento con el elemento faltante, el P, N, S y Ca mostraron ser altamente limitante al crecimiento de la cańafistula Peltophonum dibium (Duboc, 1994). Por otra parte en la Araucaria angustifolia causaron limitaciones al crecimiento de las plantas en diámetro; y el $\mathrm{Mg}$ redujo el crecimiento de las raíces (Simoes \& Couto, 1973).

El P, S y N limitaron el crecimiento en altura del cedro Cedrela fissilis; del mismo modo Jacare Piptadenia gonoacatha; del palo de hierro y de la canafistula Sena multijugla (Reno et al., 1993).

Los elementos como Ca y Mg incrementaron la biomasa aérea de las plántulas de Parica (Schizolubium amazonicum). (Lacerda et al., 1996). Las plántulas de Anacardium occidentale L son exigentes en potasio (Haag et al., 1975). Como se puede ver la ausencia de macronutrientes afecta la morfología y fisiología de las especies forestales.

Este estudio tiene como fin evaluar el efecto de la ausencia de macronutrientes en el crecimiento y nutrición de plántulas de palo de rosa Aniba rosaeodora Ducke con miras a identificar los bioelementos mas relacionados con la supervivencia de la especie.

\section{MATERIAL Y MÉTODOS}

El experimento se realizó en el vivero de producción de plántulas del Instituto Nacional de Pesquisa da Amazonía (INPA), Coordinación de Investigación en Silvicultura Tropical - INPA/CPST, localizado en los Campos del INPA V-8, en Manaus-Amazonas a (03․08' 36”S a 60 01' 19” W ) en Brasil.

Las plántulas se acomodaron en el vivero y distribuyeron en 8 tratamientos con 15 repeticiones para un total de 120 plántulas. Una muestra del suelo se utilizó para el análisis de sus propiedades químicas, la cuál se analizó en el Laboratorio Temático de Suelos y Plantas del INPA, cuyas características químicas se encuentran en la Tabla 1.

Tabla 1 - Análisis químico del suelo utilizado en el experimento, realizado en Laboratorio de Suelos y Plantas del INPA V-8.

\begin{tabular}{lc}
\hline Parâmetros & $\begin{array}{c}\text { Contenidos } \\
\mathrm{g} \mathrm{Kg}-1\end{array}$ \\
\hline $\mathrm{pH}$ (en água) & 3.79 \\
$\mathrm{pH}$ (KCL) & 3.13 \\
$\mathrm{Ca} \mathrm{cmol} / \mathrm{Kg}$ & 0.60 \\
$\mathrm{Mg} \mathrm{cmol} / \mathrm{Kg}$ & 0.14 \\
$\mathrm{~K} \mathrm{cmol} / \mathrm{Kg}$ & 0.10 \\
$\mathrm{P} \mathrm{mg} / \mathrm{Kg}$ & 8.44 \\
$\mathrm{~N} \mathrm{~g} / \mathrm{Kg}$ & 0.72 \\
$\mathrm{C} \mathrm{g} / \mathrm{Kg}$ & 14.54 \\
$\mathrm{Al} \mathrm{cmol} / \mathrm{Kg}$ & 1.10 \\
$\mathrm{M} .0 \mathrm{~g} / \mathrm{Kg}$ & 25.06 \\
\hline
\end{tabular}

Para evaluar el efecto de la deficiencia de macronutrientes en el desenvolvimiento de las plántulas de Palo-Rosa, se tomaron 15 plántulas por tratamiento y se aplicaron los siguientes tratamientos:

1) Suelo natural

2) Control (Suelo con macronutrientes)

3) Todos los macronutrientes con omisión de N.

4) Todos los macronutrientes con omisión de P.

5) Todos los macronutrientes con omisión de K.

6) Todos los macronutrientes con omisión de Ca.

7) Todos los macronutrientes con omisión de $\mathrm{Mg}$.

8) Todos los macronutrientes con omisión de $S$.

Todos los tratamientos excepto el grupo control se les suministro una solución de micronutrientes propuesta por Eira et al. (1972).

Los parámetros evaluados fueron los siguientes; materia seca de la parte aérea (MSPA), tasa de crecimiento relativo (TCR), la tasa de asimilación neta (TAN) la cual fue calculada a partir de la estimativa del área foliar. Los contenidos de macronutrientes se determinaron siguiendo la metodología 
de Silva (1999). El K, Ca, Mg y S se determinaron a través del uso del espectofotomentro de absorción atómica, el $\mathrm{P}$ en fotómetro de llama, el $\mathrm{N}$ se determinó por colorimetría (método de Nessler, titulación Kjeldhal).

El diseño experimental adoptado en este experimento fue completamente al azar con 8 tratamientos y 15 repeticiones, totalizando 120 plántulas. Los datos obtenidos se sometieron a análisis de varianza, empleando el programa Estat versión 95.

\section{RESULTADOS Y DISCUSIÓN}

\section{Crecimiento}

El crecimiento en altura de las plántulas de $A$. rosaeodora fue durante 135 días. Hubo un aumento significativo en el crecimiento en altura en los tratamientos con omisión de fósforo y potasio $(13,99$ y $12.81 \mathrm{~cm})$ en relacion al grupo control no tratado demostrando que el $\mathrm{P}$ y $\mathrm{K}$ no son limitantes para el crecimiento en altura de las plántulas (Tabla2). Este comportamiento se presenta en otras especies, por ejemplo en la jatobá (Hymenaea courbaril L.) frente a la omisión de $\mathrm{P}$ no se encontró respuesta (Duboc, 1994). En un estudio realizado por Lima (1995), encontró que la especie Tabebuia crhysotrich no fue afectada por omisión de fósforo. Por otra parte la ausencia de elementos como nitrógeno, azufre, calcio, magnesio y el grupo Control limitaron el crecimiento de la especie $A$. rosaeodora, Como se puede ver, la especie se mostró indiferente a la omisión de P y K evidenciando que la fertilización NPK no se justifica debido a que estos elementos no son muy importantes para el crecimiento inicial de $A$. rosaeodora Ducke.

El número de hojas y el área foliar de Las plantas de palo de rosa se pueden ver afectadas en suelos con baja disponibilidad de Macronutrientes. En este experimento la omisión de los elementos como N, P, K, Ca, Mg afectaron números de hojas el área foliar.

\section{Tasa de Crecimiento Relativo.}

Las plántulas de $A$. rosaeodora bajo la omisión de $\mathrm{K}$ y $\mathrm{S}$ mostraron mayor producción de MSPA (0.165 y $0.158 \mathrm{~g}^{-1} /$ día) sin diferir del tratamiento completo con $0.168 \mathrm{~g}^{-1} /$ día, Lo anterior nos indica que las plántulas con mayor tasa de crecimiento relativo (TCR) tuvieron mayor proporción de tejido meristemático el cual es el responsable del crecimiento de las plantas ya que como lo señala (Wallance, et al., 1972) las diferencias varietales en un índice fisiológico como la TCR, se deben a diferencias genéticas en los componentes que integran a la TCR; como lo son el área foliar y la tasa de asimilación neta (TAN); Entre tanto las omisiones de $\mathrm{N}$ y Ca afectaron significativamente el Crecimiento Relativo de la especie con 0.0098 y $0.0095 \mathrm{~g}^{-1} / \mathrm{dí}^{-1}$; por otro lado estos tratamientos presentaban grandes concentraciones de $\mathrm{N}$ y Ca en los tejidos foliares (Tabla 2), lo que nos indica la importancia de estos dos bioelementos para la especie,. En conclusión los tratamientos mas perjudicados tuvieron menores concentraciones de macronutrientes como N, Ca y $\mathrm{Mg}$, mientras que los mejores tratamientos obtuvieron mayor absorción de estos bioelementos.

\section{Tasa de asimilación neta}

La tasa de Asimilación Neta fue mayor en los tratamientos con omisión de S, K, P y Completo con (0.058, 0.0548, $\left.0.0544,0.0534 \mathrm{~g} / \mathrm{cm}^{2} / \mathrm{día}^{-1}\right)$ respectivamente; por otra parte los tratamientos con (suelo Natural, $-\mathrm{N},-\mathrm{Ca},-\mathrm{Mg}$ ) fueron los más perjudicados (Tabla 2), demostrando la importancia de estos bioelementos en procesos metabólicos relacionados con el aparato foliar y el tallo. Los mejores tratamientos en la TAN absorbieron mayores cantidades de $\mathrm{N}, \mathrm{Mg}$ y Ca, (Tabla 3).

Tabla 2 - Crecimiento en altura, diámetro, numero de hojas, área foliar (AF), materia seca de la parte aérea (MSPA), tasa de crecimiento relativo (TCR), tasa de asimilación neta (TAN) en plántulas de Aniba rosaeodora Ducke sometidas a la omisión de macronutrientes durante 135 días.

\begin{tabular}{|c|c|c|c|c|c|c|}
\hline Tratamientos & $\begin{array}{l}\text { Altura } \\
\text { (cm) }\end{array}$ & $\mathrm{N}^{0}$ de Hojas & $\begin{array}{l}\text { Área F } \\
\left(\mathrm{cm}^{2}\right)\end{array}$ & $\begin{array}{c}\text { MSPA } \\
(\mathrm{g})\end{array}$ & $\begin{array}{l}\text { TCR } \\
\text { g/día }\end{array}$ & $\begin{array}{c}\text { TAN g. } \mathrm{cm}^{2} \text { /día } \\
\text { Transf. } \\
\text { arc-sen }\end{array}$ \\
\hline Suelo natural & $9.5 b$ & $6.5 b$ & $9.45 b$ & $0.79 \mathrm{~b}$ & $0.88 a b$ & $0.03007 \mathrm{~b}$ \\
\hline Control & 12.06ab & $8.5 b$ & $22.6 \mathrm{a}$ & $2.1 \mathrm{a}$ & $0.168 \mathrm{a}$ & $0.0534 a$ \\
\hline$-\mathrm{N}$ & $9.8 \mathrm{~b}$ & $9.75 a b$ & $18.9 a b$ & $1.51 a b$ & $0.098 \mathrm{~b}$ & $0.0479 a b$ \\
\hline$-P$ & $13.9 a$ & $10 a b$ & 16.31ab & $1.53 a b$ & $0.108 a b$ & $0.0544 a$ \\
\hline$-K$ & $12.81 \mathrm{a}$ & $9 a b$ & 17.59ab & $1.92 \mathrm{a}$ & $0.158 \mathrm{a}$ & $0.0548 a$ \\
\hline$-\mathrm{Ca}$ & $11.62 a b$ & $10 a b$ & 16.79ab & $1.38 a b$ & $0.095 b$ & $0.049 a b$ \\
\hline$-M g$ & $11.8 \mathrm{ab}$ & $9 a b$ & 17.71ab & $1.53 a b$ & $0.108 a b$ & $0.0511 a$ \\
\hline$-S$ & 11.61ab & $12.75 a$ & $23.1 \mathrm{a}$ & $2.02 \mathrm{a}$ & $0.165 a$ & $0.0581 a$ \\
\hline $\mathrm{F}$ & $3.04^{* \star}$ & $4.57^{\star *}$ & $3.44^{* *}$ & $4.27^{\star \star}$ & $2.48^{\star \star}$ & $4.13^{* \star}$ \\
\hline DMS (5\%) & 3.665 & 3.82 & 11.96 & 0.9572 & 0.101 & 0.0194 \\
\hline CV (\%) & 27.89 & 17.17 & 6.29 & 25.52 & 27.85 & 16.6 \\
\hline
\end{tabular}

* Significativo en la Test $\mathrm{F}$ al nivel $5 \%$ de probabilidad. 
Para el N un rango de $\left(17.43\right.$ y $\left.19.11 \mathrm{~g} / \mathrm{Kg}^{-1}\right)$ y $\mathrm{Mg}$ un rango de $\left(2,02\right.$ y $\left.2,7 \mathrm{~g} / \mathrm{Kg}^{-1}\right)$ y Ca $\left(4\right.$ y $\left.8,2 \mathrm{~g} / \mathrm{Kg}^{-1}\right)$. Sin embargo, el tratamiento sin azufre absorbió mayores concentraciones de estos elementos. Por otra parte la omisión de P permitió una buena absorción de macronutrientes $(\mathrm{Ca}, \mathrm{Mg}$ y $\mathrm{K}$ ) sobre todo en el aparato fotoasimilador y no perjudico la TAN. La omisión de fósforo perjudico la TCR de la planta en términos de tiempo. Estos datos sugieren que los tratamientos $-\mathrm{K},-\mathrm{S}$, completo, asimilaron mejor $\mathrm{CO}_{2}$ y nutrientes como Ca y $\mathrm{Mg}$ son importantes en la producción de MSPA (Tabla 2).

\section{NUTRICION MINERAL}

La deficiencia de nitrógeno afecto todo el crecimiento de la parte aérea (MSPA) y la tasa de crecimiento relativo (TCR). El tratamiento con omisión de $S$ presento mayor contenido de nitrógeno (Tabla 3), este tratamiento también presento un aumento significativo de biomasa aérea, de TAN y de TCR (Tabla 2). La reducción del crecimiento proporcionada por la deficiencia de nitrógeno es resultado de las funciones que el nutriente desempeña en la planta. Normalmente el $\mathrm{N}$ es el nutriente mas exigido una vez actúa en las moléculas de los aminoácidos, proteínas, enzimas y productos secundarios (Marschner, 1995).

El tratamiento completo y con omisión de $\mathrm{Mg}$ permitió mayor absorción de fósforo; del mismo modo, el tratamiento completo produjo mayor MSPA y absorbió grandes cantidades de $\mathrm{P}\left(1.45 \mathrm{~g} / \mathrm{Kg}^{1}\right)$.

El tratamiento con omisión de fósforo obtuvo el mayor crecimiento en altura con una concentración de $(0.81 \mathrm{~g}$ $/ \mathrm{Kg}^{1}$ ) al parecer las plántulas de $A$. rosaeodora tienen un bajo requerimiento nutricional de $\mathrm{P}$ para el crecimiento en altura confirmando que la planta esta adaptada a suelos ácidos. El fósforo no es un elemento importante para el crecimiento de la planta $A$ rosaeodora; inclusive Marschner (1991) afirma que este elemento es poco disponible en suelos ácidos como los del Amazonas; y una característica de adaptación de las planta a estos suelos es el crecimiento normal de la planta en poca disponibilidad de este elemento. Por otra parte en la ciudad de Manaus-Am., se realizo un experimento en donde se analizaron plantas de A. rosaeodora en las edades de 3 y 5 años en plantíos a plena luz a un espaciamiento de $3 \times 2 \mathrm{M}$ en un área de $30.000 \mathrm{~m}^{2}$; se encontró diferencias significativas en la bases trocables $\mathrm{Mg}, \mathrm{K}, \mathrm{P}$ entre ramas y hojas, además se demostró que las muestras de suelo de esta parcela presentaban una acidez pronunciada, la cual no perjudicó la absorción de nutrientes (Takeda, S. P, 2008).
Los contenidos de potasio en las hojas palo de rosa bajo omisión de este elemento no difiere del contenido encontrado en el tratamiento completo (Tabla 3). Sugiriendo que las plántulas de palo de rosa poseen un elevada capacidad de extracción de potasio del substrato, del mismo modo, presenta un bajo requerimiento fisiológico. La absorción de potasio fue significativamente favorecida en el tratamiento con omisión de $\mathrm{Ca}$, probablemente este acontecimiento se debe a la competencia que tiene el K con el Ca por el misma vía de absorción-apoplastos (Malavolta, 1989). Además de esto es importante mencionar que las plántulas del tratamiento con omisión de K y el tratamiento completo absorbieron menores concentraciones de $\mathrm{K}$ (Tabla 3) y tuvieron mayor MSPA cuando comparadas con omisiones de (N, P, Mg y Ca) (Tabla 2). A este fenómeno no se le encontró referencia en la literatura que explicara el motivo por el cual las plántulas que absorbieron menos $\mathrm{K}$ tuvieran mayor MSPA.

Para la especie $A$. rosaeodora los elementos como calcio y magnesio fueron muy importantes para la producción MSPA, tasa de crecimiento relativo (TCR) y la tasa de asimilación neta (TAN) (Tabla 2) y además fueron los nutrientes más absorbidos (Tabla 3). Nuestros datos sugieren que la especie es calcifica y el $\mathrm{Mg}$ es bioelemento importante para el aumento significativo de área foliar e índice fotosintético.

Según Marschner, (1991) en las plantas silvestres adaptadas a suelos ácidos las concentraciones de $\mathrm{Ca}$ o $\mathrm{Mg}$ son altas, indicando adaptación a este suelo para evitar el estrés. La especie A rosaeodora obtuvo concentraciones altas de estos elementos (Tabla 3).

De un modo general el requerimiento de las plantas para el S acompańa la demanda de P (Malavolta, 1980); entre tanto, en la Tabla 3 se puede observar que la absorción de $S$ fue, aproximadamente 2 veces menor que la absorción de $\mathrm{P}$ indicando que la exigencia nutricional de $A$. rosaeodora por el $S$ es inferior a la del $P$.

Las mayores concentraciones de nutrientes en las hojas son comunes en la mayoría de las especies forestales, debido a la gran actividad metabólica de este órgano, entre tanto, las concentraciones de nutrientes disminuyen y la calidad del aceite esencial empeora a medida que las hojas envejecen. Del mismo modo los factores genéticos y ambientales también son controladores de los contenidos minerales en los vegetales (Maffeis, et al., 2000). 
Tabla 3 - Contenido de macronutrientes de la materia seca de las hojas de plántulas de palo de rosa Aniba rosaeodora ducke sometidas a tratamientos con omisión de macronutrientes. Laboratorio de Suelos y Plantas, CPST- INPA.

\begin{tabular}{|c|c|c|c|c|c|c|}
\hline \multirow[t]{2}{*}{ Tratamientos } & \multicolumn{6}{|c|}{$\begin{array}{l}\text { Contenido de nutrientes } \\
\qquad \mathrm{g} \mathrm{Kg}^{1}\end{array}$} \\
\hline & $\mathrm{N}$ & $P$ & $\mathrm{~K}$ & $\mathrm{Ca}$ & $\mathrm{Mg}$ & $S$ \\
\hline Suelo natural & $16.22 b$ & $1.02 a b$ & $9.22 a b$ & $3.03 b$ & 1.22ab & $0.65 a$ \\
\hline Control & 17.71ab & $1.45 a$ & $8.67 \mathrm{~b}$ & $8.26 a$ & $2.27 a$ & $0.50 \mathrm{~b}$ \\
\hline$-\mathrm{N}$ & $15.65 b$ & $1.12 a b$ & $9.92 a b$ & $4.69 a$ & $2.36 a$ & $0.30 d$ \\
\hline$-P$ & 17.43ab & $0.85 b$ & 10.22ab & $4.06 a$ & $1.81 a$ & $0.30 d$ \\
\hline$-K$ & 18.58ab & $1.03 a b$ & 8.96b & $4.76 \mathrm{a}$ & $2.30 \mathrm{a}$ & $0.20 f$ \\
\hline$-\mathrm{Ca}$ & 17.19ab & $1.03 a b$ & $12.59 a$ & $2.48 b$ & $2.02 a$ & $0.28 \mathrm{de}$ \\
\hline$-M g$ & 18.79ab & $1.41 \mathrm{a}$ & $11.04 a b$ & $4.3 a$ & $0.82 b$ & $0.36 c$ \\
\hline$-S$ & $19.11 \mathrm{a}$ & $1.02 a b$ & $9.95 a b$ & $6.88 \mathrm{a}$ & $2.7 \mathrm{a}$ & $0.25 \mathrm{e}$ \\
\hline $\mathrm{F}$ & 2.54 & $3.56^{\star \star}$ & $2.51^{\star}$ & $6.31^{* *}$ & $5.47 * \star$ & $286^{\star \star *}$ \\
\hline CV (\%) & 3.07 & 18.79 & 15.87 & 20.99 & 27.69 & 4.95 \\
\hline
\end{tabular}

* Significativo en la Test $\mathrm{F}$ al nivel $5 \%$ de probabilidad.

\section{BIBLIOGRAFIA CITADA}

Alencar, J.C.; Fernandes, N.P. 1978. Desenvolvimento de árvore nativa em ensaios de espécies. 1 .pau-rosa (Aniba duckei Kostermans). Acta Amazônica 8 (4):523-541.

Calderon, E. 1997. Lista de plantas Colombianas en peligro. Versión July 1997. Instituto de Investigación de recursos Biológicos Alexander von Humbolt. Bogota-Colombia, 14 pp

Carvalho, J.O.P. 1983. Abundância, freqüência e grau de agregação do pau-rosa (Aniba duckei Kostermans) na floresta nacional do Tapajós. Boletim de Pesquisa 53 : 18-24

Duboc, E. 1994. Requerimentos nutricionais de espécies nativas: Hymenaea cuorbari L var. stilbocarpa (HAYNE) LEE ET LANG. (jatoba), Copaifera langsdarffi DESF (OLEO CAPAIBA) e Peltophorum dubium (SPRENG) TAUB .( Canafistula). Lavras. ESAL. Tesis de Maestria en manejo ambiental.Belo Horizonte, Minas Gerais. 128pp.

Eira, P.A.; Almeida, D.I.; Silva, W.C. 1972. Fatores nutricionais Iimitantes do desenvolvimento de três leguminosas forrageiras em solo podozólico vermelho amarelo. SER. ARGON. Pesq. Agropecuária 7 :185-192.

Haag, H.P.; Sarruge, J.R.; Oliveira, G.D.; Dechen, A.R. 1975. Nutriçáo mineral do cajueiro (Anacardium occidentale L). Anais da E.S.A. Luiz de Queiroz 32: 185-190

IBAMA. 1992. Portaria No 37. Diário Oficial da União de 3 de abril de 1992. Brasília, DF.

Lacerda, M. P.C.; Motta, P.E; Lanza, T.C.L.; Carvalho, J.G. 1996. Avaliaçáo dos teores de $\mathrm{Ca}, \mathrm{Mg}$ e $\mathrm{S}$ em parica (Schizolobium amazonicum) em tratamentos em solução nutritiva com omissão de macronutrientes. Anais da E.S.A. Luiz de Queiroz 32, Belo Horizonte, Minas Gerais. 359pp.

Lima, H.N. 1995. Crescimento inicial de sete espécies arbóreas nativas em resposta à adubação com NPK a campo. Tesis de Maestria.: Universidade Federal de Lavras. 53pp.
Maffeis, A.R.; Silveira, R.L.V.A .; Brito, J.O. 2000. Reflexos das deficiências de macronutrientes e Boro no crescimento de plantas, produçáo e qualidade de óleo essencial em (Eucalyptus citriodora), Scientia florestalis 57: 87-98.

Malavolta, E. 1980. Elementos de nutrição mineral de plantas. São Paulo, Editora Agronómica Ceres. 251 pp.

Malavolta, E.; Vitti, G.C.; Oliveira, S.A. 1989. Avaliação do estado nutricional das plantas: Princípios e aplicaçóes. Piracicaba- SP. $201 \mathrm{pp}$.

Marschner, H. 1991. Mechanism of adaptation of plants to acids soil. Plant and soil 134(1):1-20.

Marschner H., 1995. Mineral nutrition of higher plants. Second Edition. Institute of plant Nutrition University of Hohenheim, Germany. 889 pp.

Renó, N.B.; Vale, F.R.; Curi, N.; Siquera, J.O. 1993. Requerimentos nutricionais de quatro espécies florestais nativas En: CONGRESO BRASILEIRO DE CIENCIA DO SOLO 24, Goiânia, En: Resumos, Goiânia: SBCS, p.211-212.

Sampaio, P.T.B. 1987. Propagaçáo vegetativa do pau-rosa (Aniba rosaeodora Ducke) pelo método a estaquia. Tesis de Maestria (Manejo forestal), Instituto Nacional de Pesquisas da Amazônia / Fundação Universidade do Amazonas. Manaus, Amazonas. 94pp

Sampaio, P. T. B. 1999. Pau-rosa (Aniba rosaeodora Ducke). In: Paulo de Tarso B. Sampaio; Charles R. Clement. (Org.). 1999. Biodiversidade Amazônica: exemplos e estratégias de utilização Vol 11, 1 ed. SEBRAE, Manaus, AM. p. 290-297

Silva, F.C. 1999. Extração de elementos quimicos do tecido vegetal. Manual de análises químicas de solos, plantas e fertilizantes. EMBRAPA. Brasília. 223pp.

Simoes J.W.;Couto, H.T.Z. 1973. Efeitos da omissão de nutrientes na alimentação mineral do pinheiro do Paraná Araucária angustifólia (Bert) O. Ktze cultivado em vasos. IPEF, Piracicaba 7 :3-40 
Takeda, P. S. 2008. Avaliação de biomassa e óleo de rebrotas de galhos e folhas de pau-rosa (Aniba rosaeodora Ducke) em plantios comerciais submetidos à poda e adubaçáo. Tesis de Maestria (Ciencias de Florestas Tropicales). Instituto Nacional de Pesquisas da Amazônia/Universidade Federal do Amazonas. Manaus, Amazonas. 73pp.

Viera, A.N. 1970. Aspectos silviculturais do pau-rosa (Aniba rosaeodora duckei Kostermans) II- Estudos sobre métodos de propagação. Acta Amazonica, 2 (1) :51-58.
Wallace, D.H.; Ozbun, L.J. \&. Munger, M.H. 1972. Physiological genetics of crop yield. Advan. Agron 24:1- 96

Werkhoven, M.C.M. 1997. Threatened trees of Suriname, A list compiled for the WCMC / SSC Conservation and Sustainable Management of trees project. 382 pp.

Recebido em 16/01/2009

Aceito em 05/01/2010 